\title{
Liaison cuivre-alumine : évolution et caractérisation microscopique des composés interfaciaux
}

\author{
Catherine Beraud et Claude Esnouf
}

Groupe de Métallurgie Physique et de Physique des Matériaux, Bât. 502, INSA de Lyon (URA 341), 69621 Villeurbanne cedex, France

(Reçu le 6 février 1990, accepté le 26 mars 1990)

Résumé. - La thermocompression et la méthode de l'eutectique gaz-métal $\mathrm{Cu}-\mathrm{O}$ en atmosphère neutre, ont été mises en ouvre pour effectuer la liaison entre le cuivre et l'alumine polycristalline. Les observations en Microscopie Electronique en Transmission de la région interfaciale révèlent l'exsitence d'un composé intermédiaire, l'oxyde mixte $\mathrm{CuAlO}_{2}$, oxyde qui se destabilise au bénéfice d'une couche d'alumine pour des maintiens en température de longue durée. L'orientation cristallographique de cet oxyde est bien adaptée pour pallier aux écarts de dilatation, comme en témoigne la caractérisation microscopique de l'oxyde mixte.

\begin{abstract}
Bonding between copper and polycrystalline alumina has been obtained by hot pressing of the two materials under low pressure and by the $\mathrm{Cu}-\mathrm{Cu}_{2} \mathrm{O}$ eutectic method in a protective atmosphere. The observation of bonding zone by means of Transmission Electron Microscopy reveals the presence of an interfacial compound : a binary oxide $\mathrm{CuAlO}_{2}$ which is destabilized into alumina by annealing for long time. A microscopic characterization of the binary oxide shows that it is well suited for adaptation of the thermal expansion.
\end{abstract}

\section{Introduction.}

Les potentialités offertes par l'association d'un métal avec une céramique suscitent un large intérêt depuis de très nombreuses années (on en veut pour preuve l'utilisation des émaux dès l'antiquité...). Cependant c'est seulement avec l'apparition des céramiques techniques que l'intérêt porté à la liaison entre un métal et une céramique s'est vraiment affirmé.

Essentiellement, trois domaines d'application sont distingués [1] :

Applications à l'électronique avec la réalisation de tubes, isolateurs, condensateurs, jonctions.... Les matériaux utilisés sont le plus souvent l'alumine liée au cuivre ou au platine.

Applications bio-médicales avec la réalisation de prothèses. Naturellement l'emploi d'un métal noble et biologiquement inerte est nécessaire comme l'or, l'argent, la platine. Le champ 
d'application couvre la fabrication de prothèses dentaires ainsi que tous les organes mécaniques et électriques destinés à être implantés dans le corps humain.

Applications de construction. Les propriétés intéressantes des céramiques thermomécaniques (PSZ, $\mathrm{Si}_{3} \mathrm{~N}_{4}, \mathrm{SiC}, \mathrm{Al}_{2} \mathrm{O}_{3} \ldots$ ) telles que leur tenue à haute température, leur résistance à la corrosion et à l'usure, leur faible conduction thermique et électrique, ne sont véritablement exploitées dans une structure que sous la forme d'assemblage avec des parties métalliques faciles à mettre en forme. C'est ainsi que l'on a songé à la technologie du dépôt céramique sur métal en vue de réaliser une barrière thermique dans les turbines à gaz et les moteurs thermiques. Mais bien d'autres applications sont exploitées.

Les techniques de réalisation des couples sont nombreuses mais on peut distinguer deux classes de procédés : liaisons par passage à l'état liquide et liaison à l'état solide.

La première concerne les techniques d'émaillage (dépôt céramique contenant des fondants et des opacifiants), de brasage après métallisation de la céramique (procédé au molybdènemanganèse datant de 1950).

Dans la seconde, il y a lieu de séparer les procédés basés sur un accrochage purement mécanique (forgeage, assemblage par explosion, scellement par friction, projection plasma), de ceux dits de soudure réactive. Dans une atmosphère et à une température adaptées, un contact entre les deux surfaces à lier est assuré de sorte à développer des processus diffusionnel et réactionnel capables d'assurer une bonne tenue mécanique. Un grand nombre de couples métal/céramique ont été ainsi élaborés. Si une pression uniaxiale souvent très variable, est exercée sur les antagonistes, on dit que l'on a affaire à la technique de thermocompression HP (Hot Pressed). Lorsqu'une pression isostatique est utilisée, c'est la technique HIP (Hot Isostatic Pressure) plus récente. Le tableau I récapitule les différents couples étudiés en excluant les liaisons verre/métal ou obtenues par la méthode de la goutte fondue (mesure de la mouillabilité) (sur ce point, se reporter aux références $[2,3]$ ). Bon nombre de résultats se rapportent à la liaison alumine/métal obtenue par thermocompression et au couple nitrure de silicium/acier pour ce qui est de la technique HIP.

En ce qui concerne la caractérisation des liaisons, le test mécanique reste largement prédominant comme il se doit. L'approche microscopique tend à se développer ; elle nous concerne totalement dans la présente contribution. L'intérêt de la microscopie à transmission pour l'étude de la liaison, est d'apporter une information très locale dans la zone interfaciale (morphologie, nature des composés, défauts...). A terme ces observations vont nous permettre de détailler les processus intervenus au cours de l'élaboration.

\section{Position du problème.}

Contrairement à la soudure des métaux et alliages, la réalisation de joints métal/céramique semble difficile en raison du contraste de leurs propriétés électriques, thermiques, mécaniques et chimiques. Si on compare les courbes de Gordon-Morse relatives à l'énergie potentielle atomique dans un métal et une céramique, on remarque un puits de potentiel profond dans le cas de la céramique, indicateur d'un haut point de fusion et une faible valeur du coefficient d'autodiffusion. Ce puits est également plus étroit signifiant un haut module d'élasticité et un faible coefficient de dilatation thermique.

Le tableau II regroupe des valeurs prises par quelques grandeurs physiques associées au cuivre et à l'alumine, couple qui se révèle assez moyen par rapport au comportement général.

Ajoutons qu'il est admis que l'organisation atomique en surface des oxydes est assez différente de celle du massif. Il se produit une rétraction des cations de sorte que seuls les anions sont présents en surface [32]. On conçoit dès lors la difficulté d'allier un métal à une céramique, le nuage électronique du composant métallique tend à repousser les charges négatives portées par 
Tableau I. - Couples métal/céramique étudiés. Elaboration : HP : thermocompression, HIP : pressage isostatique. Analyse: TEM, SEM : microscopie à transmission et balayage, HREM : microscopie haute résolution, $\mu X$ : microanalyse $X, R$ : réflectivité. Essais: Mec : mécanique, $H_{v}$ : dureté.

[Studied metal/ceramic systems. Elaboration: HP: hot pressing, HIP: hot isostatic pressure. Analyse: TEM, SEM: transmission and scanning electron microscopy, HREM: high resolution electron microscopy, $\mu \mathrm{X}$ : X microanalysis, R: reflectivity. Tests: Mec: mechanics, $H_{v}$ : hardness.]

\begin{tabular}{|c|c|c|c|c|c|c|c|c|c|c|c|c|c|c|c|}
\hline Mé/Cér. & $\mathrm{Al}$ & $\mathrm{Ti}$ & $\mathrm{Cr}$ & $\mathrm{Fe}$ & $\mathrm{Ni}$ & $\mathrm{Cu}$ & $\mathrm{Zr}$ & $\mathrm{Nb}$ & Pd & $\mathrm{Ag}$ & $\mathrm{Ta}$ & w & $\mathbf{P t}$ & $\mathrm{Au}$ & $\mathbf{P b}$ \\
\hline $\mathrm{Al}_{2} \mathrm{O}_{3}$ & \begin{tabular}{|l|} 
HP \\
R, HREM \\
Mec \\
$4,5,17$. \\
22
\end{tabular} & \begin{tabular}{|l|}
$\mathrm{HP}$ \\
SEM, $\mu \mathrm{X}$ \\
$\mathrm{Hv}$ \\
13,20
\end{tabular} & \begin{tabular}{|l}
$\mathrm{HP}$ \\
$\mu \mathrm{X}$ \\
$\mathrm{Mec}$ \\
11
\end{tabular} & \begin{tabular}{|l|}
$\mathrm{HP}, \mathrm{HIP}$ \\
SEM, $\mu \mathrm{X}$ \\
$\mathrm{Mec}$ \\
$4,26,27$ \\
\end{tabular} & \begin{tabular}{|l|} 
HP, HIP \\
SEM, $\mu \mathrm{X}$ \\
Mec, Hv \\
$4,7,11$
\end{tabular} & \begin{tabular}{|l|} 
HP \\
TEM, $\mu \mathrm{X}$ \\
Mec \\
$4,6,15$, \\
16,17 \\
18,19, \\
21
\end{tabular} & & \begin{tabular}{|l|} 
HP \\
HREM, $\mu \mathrm{X}$ \\
Mec, Hv \\
$12,13,14$, \\
27
\end{tabular} & $\begin{array}{l}\mathrm{HP} \\
\mu \mathrm{X} \\
7\end{array}$ & & $\begin{array}{l}\mathrm{HP} \\
\mathrm{R} \\
13,17 \\
\end{array}$ & & $\begin{array}{l}\text { HP } \\
\text { TEM } \\
\text { Mec } \\
4,7,8, \\
9,10,21\end{array}$ & $\begin{array}{l}\text { HP } \\
\text { SEM } \\
\text { Mec } \\
7.8\end{array}$ & 4 \\
\hline $\mathrm{Si}_{3} \mathrm{~N}_{4}$ & $\begin{array}{l}\mathrm{HP} \\
\mathrm{Mec} \\
27\end{array}$ & & & \begin{tabular}{l|} 
HP, HIP \\
TEM, $\mu \mathrm{X}$ \\
Mec \\
23,24, \\
25
\end{tabular} & & & $\begin{array}{l}\text { HP } \\
\text { SEM } \\
13\end{array}$ & & & & & $\begin{array}{l}\text { HIP } \\
\text { Mec } \\
25\end{array}$ & & & \\
\hline $\begin{array}{l}\text { AlN } \\
\text { TiN } \\
\text { BN }\end{array}$ & & & & \begin{tabular}{l|} 
HP, HIP \\
SEM, $\mu \mathrm{X}$ \\
Mec \\
23,24, \\
26
\end{tabular} & & & & & & & & & & & \\
\hline $\begin{array}{l}\mathrm{TiC} \\
\mathrm{TaC}\end{array}$ & & & & $\begin{array}{l}\text { HP } \\
\text { SEM } \\
\text { Mec } \\
13.26\end{array}$ & & & & & & & & & & & \\
\hline $\mathrm{SiC}$ & $\begin{array}{l}\text { HP } \\
\text { HREM } \\
6,28\end{array}$ & & & & & & & $\begin{array}{l}\text { HP } \\
13\end{array}$ & & & & & & & \\
\hline $\mathrm{Y}_{2} \mathrm{O}_{3}$ & & & & & & & & \begin{tabular}{|l|}
$\mathrm{HP}$ \\
13
\end{tabular} & & & & & & & \\
\hline $\mathrm{MgO}$ & & & & & \begin{tabular}{|l|}
$\mathrm{HP}$ \\
$\mu \mathrm{X}$ \\
$\mathrm{Mec}$ \\
7,8 \\
\end{tabular} & & & & \begin{tabular}{|l}
$\mathrm{HP}$ \\
$\mu \mathrm{X}$ \\
7
\end{tabular} & & & & $\begin{array}{l}\mathrm{HP} \\
\mathrm{Mec} \\
7.8 \\
\end{array}$ & & \\
\hline $\mathrm{BeO}$ & & & & & $\begin{array}{l}\mathrm{HP} \\
\mathrm{Mec} \\
7\end{array}$ & & & & & & & & \begin{tabular}{|l} 
HP \\
SEM, $\mu \mathrm{X}$ \\
Mec \\
7 \\
\end{tabular} & & \\
\hline $\mathrm{ZrO}_{2}$ & $\begin{array}{l}\mathrm{HP} \\
\text { TEM } \\
\mathrm{Hv} \\
29 \\
\end{array}$ & & & & & & & & & HP & & & \begin{tabular}{|l} 
HP \\
SEM, $\mu \mathrm{X}$ \\
7.8 \\
\end{tabular} & & \\
\hline
\end{tabular}

les atomes d'oxygène polarisés de la surface. Un tel état de fait se matérialise par un fort angle de mouillage entre les métaux liquides et les oxydes. Cette manifestation macroscopique est révélatrice de phénomènes physiques à l'interface. Ces aspects sont quantifiés par une grandeur appelée travail d'adhésion $W$ donnée par la somme des énergies superficielles du métal et de la céramique diminuée de l'énergie interfaciale :

$$
W=\gamma_{\mathrm{m}}+\gamma_{\mathrm{c}}-\gamma_{\mathrm{mc}}
$$

Elle représente le gain d'énergie produit au moment du recouvrement d'un matériau par un autre. Le travail d'adhésion est obtenu à partir de la relation d'Young-Dupré :

$$
W=\gamma_{m}(1+\cos \Theta)
$$


Tableau II. - Caractéristiques physiques du cuivre et de l'alumine. ( $\Delta H_{\mathrm{f}}^{\circ}$ : enthalpie de formation de la liaison métal-oxygène ; $\Delta H_{\mathrm{M}[\mathrm{M}]}$ : enthalpie de liaison métal-métal (voir [33])).

[Physical features of copper and alumina $\left(\Delta H_{\mathrm{f}}^{\circ}\right.$ : enthalpy of formation of the M metal oxide, $\Delta H_{\mathrm{M}[\mathrm{M}]}$ : enthalpy of mixing of a metal into another metal (see [33])).]

\begin{tabular}{|c|c|c|c|c|c|c|c|c|}
\hline & $\begin{array}{c}T_{\mathrm{f}} \\
\left({ }^{\circ} \mathrm{C}\right)\end{array}$ & $\begin{array}{c}\text { Coeff. } \\
\text { dilatation } \\
\left({ }^{\circ} \mathrm{C}^{-1}\right)\end{array}$ & $\begin{array}{c}\text { Module } \\
\text { Young } \\
(\mathrm{GPa})\end{array}$ & $\begin{array}{c}\text { Conduct. } \\
\text { therm. } \\
(\mathrm{Wm} / \mathrm{s})\end{array}$ & $\begin{array}{c}\text { Coeff. } \\
\text { diffusion } \\
\left(\mathrm{cm}^{2} / \mathrm{s}\right)\end{array}$ & $\begin{array}{c}\text { Energie } \\
\text { surface } \\
\left(\mathrm{mJ} / \mathrm{m}^{2}\right)\end{array}$ & $\begin{array}{c}\Delta H_{\mathrm{f}}^{\circ} \\
\left(20^{\circ} \mathrm{C}\right) \\
(\mathrm{kJ} / \mathrm{at} \mathrm{O})\end{array}$ & $\begin{array}{c}\Delta H_{\mathrm{M}[\mathrm{M}]} \\
(\mathrm{kJ} / \mathrm{mole})\end{array}$ \\
\hline $\mathrm{Cu}$ & 1083 & $17 \times 10^{-6}$ & 125 & 153 & $\begin{array}{c}2 \times 10^{-9} \\
\left(\mathrm{a} 1000^{\circ} \mathrm{C}\right)\end{array}$ & $\begin{array}{c}1200 \\
\left(\mathrm{à} 1000^{\circ} \mathrm{C}\right)\end{array}$ & $\begin{array}{c}\mathrm{Cu}_{2} \mathrm{O} \text { : } \\
-195,4\end{array}$ & $\begin{array}{c}\mathrm{Al}[\mathrm{Cu}] \\
-36\end{array}$ \\
$\mathrm{Al}_{2} \mathrm{O}_{3}$ & 2037 & $6,5 \times 10^{-6}$ & 340 & 1,4 & $\begin{array}{c}\mathrm{Al}: 10^{-19} \\
\mathrm{O}: 10^{-23} \\
\left(\mathrm{a} 1000^{\circ} \mathrm{C}\right)\end{array}$ & $\begin{array}{c}\text { mono } \\
748[30] \\
\text { poly } \\
1560[31] \\
\left(\mathrm{à} 1000^{\circ} \mathrm{C}\right)\end{array}$ & $\begin{array}{c}\mathrm{Al}_{2} \mathrm{O}_{3} \\
-565,8\end{array}$ & --- \\
& & & & & &
\end{tabular}

où $\Theta$ est l'angle de contact à l'équilibre entre le métal et la céramique. Les grandeurs $\gamma_{\mathrm{m}}$ et $\Theta$ sont, le plus souvent, mesurées par la méthode de la "goutte posée" (Fig. 1). Cette technique ancienne demande quelques précautions analysées en detail par Eustathopoulos et al. [par ex. 33] qui développe l'idée que la valeur du travail d'adhésion résulte à la fois des intéractions métaloxygène et métal-métal dans le cas des couples métal/alumine. La figure 2 donne les valeurs expérimentales du travail d'adhésion de certains métaux sur l'alumine, en fonction de cette même grandeur calculée par la relation :

$$
W=\left(\alpha \Delta H_{\mathrm{f}}^{\circ}+\beta \Delta H_{\mathrm{Al}[\mathrm{Me}]}\right) \Omega^{-1}
$$

$\Omega$ : surface couverte à l'interface par l'espèce métallique, $\alpha$ et $\beta$ des constantes.

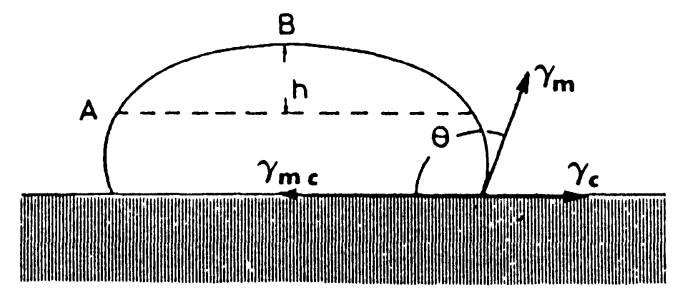

Fig. 1. - Goutte posée de métal liquide sur une céramique.

[Sessile drop of a liquid on a ceramic.]

L'étude des intéractions entre les premières couches des deux constituants demande l'emploi de techniques fines d'analyse de surface (AES, LEED, SIMS,...), couramment mises en œuvre dans l'étude des couches minces déposées [34].

Dans un tel contexte, il est certain que la troisième composante que constitue l'atmosphère, a une grande importance, spécialement dans le cas des atmosphères oxydantes ou réductrices. Un 


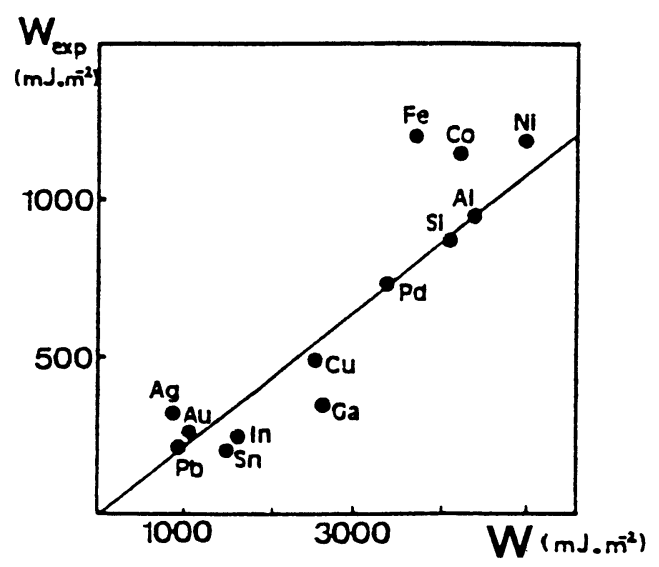

Fig. 2. - Valeurs expérimentales du travail d'adhésion sur l'alumine en fonction des valeurs calculées par la relation (3) [33].

[Experimental values of the adhesion work of different metals on alumina as a function of calculated values obtained by the relation (3) [33].]

effet tensioactif de l'oxygène a été observé, dans le cas du couple cuivre-alumine, par Chaklader et al. [35]. Par des ajouts de $\mathrm{CuO}$ dans le cuivre déposé sur de l'alumine, ces auteurs observent une très forte diminution de l'angle de contact passant très nettement en-dessous de $90^{\circ}$, limite de mouillabilité.

La même équipe a repris ces mesures mais cette fois en atmosphère composée d'un mélange $\mathrm{CO}$ et $\mathrm{CO}_{2}$ [36]. La figure 3 présente l'évolution de $\Theta, \gamma_{\mathrm{Cu}}$ et $\gamma_{\mathrm{mc}}$ à $1200^{\circ} \mathrm{C}$ en fonction de la concentration en oxygène dans le cuivre. La valeur choisie pour l'énergie superficielle de l'alumine est de $748 \mathrm{~mJ} / \mathrm{m}^{2}$. Ces évolutions ont pour conséquence de faire varier le travail d'adhésion qui passe d'une valeur autour de $400 \mathrm{~mJ} / \mathrm{m}^{2}$ pour $10^{-6}$ at $\% \mathrm{O}^{2-}$ à environ $900 \mathrm{~mJ} / \mathrm{m}^{2}$ pour $10^{-2}$ at $\%$ $\mathrm{O}^{2-}$; elle décroît ensuite légèrement pour de plus fortes concentrations [36].

Un effet similaire a été observé avec d'autres métaux comme le nickel, le fer [37], l'étain [38] et l'argent $[37,38]$. Egalement, certaines espèces métalliques ont un effet tensioactif à l'interface $\mathrm{Cu} / \mathrm{Al}_{2} \mathrm{O}_{3}$; citons l'aluminium [39] ou le titane [3] dissous dans le cuivre. En conséquence on conçoit la difficulté d'atteindre la mouillabilité intrinsèque, d'autant que la surface des supports oxyde est susceptible d'évoluer en fonction du temps et de l'atmosphère [40] (lacunes d'oxygène et mise en ordre). La tension superficielle de l'oxyde $\gamma_{c}$ et par là-même le travail d'adhésion s'en trouvent changés.

En résumé, l'angle vrai de contact du cuivre sur l'alumine est très élevé ; il conduit à une faible valeur du travail d'adhésion. Ces valeurs sont données par les relations suivantes : $\Theta=$ $131^{\circ}-0,01^{\circ}(T-1373)$ et $W=441+0,1(T-1373) \mathrm{mJ} / \mathrm{m}^{2}$ [39]. La mouillabilité intrinsèque de l'alumine par le cuivre n'est pas bonne. Cependant un apport d'oxygène dans le cuivre l'améliore considérablement.

Dans les expériences qui vont être décrites ensuite, nous avons délibérément choisi la voie qui consiste à apporter de l'oxygène à l'interface cuivre/alumine par une oxydation superficielle préalable du cuivre, l'atmosphère au moment de la liaison restant neutre (balayage d'argon). 


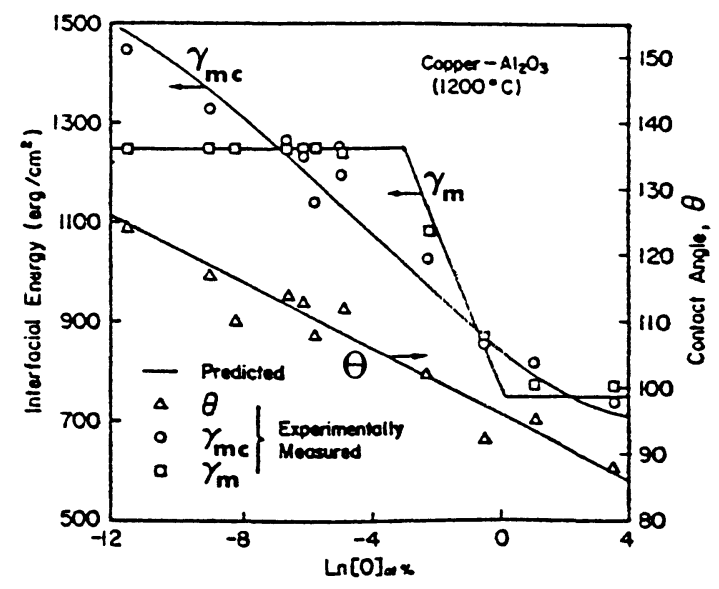

Fig. 3. - Evolution de $\Theta, \gamma_{\mathfrak{c}}, \gamma_{\mathrm{mc}}$ pour le couple $\mathrm{Cu}$ /alumine [37].

[Evolution of $\mathrm{O}, \gamma_{\mathfrak{c}}, \gamma_{\mathrm{mc}}$ in the case of copper/alumina system [37].]

\section{Appareillage et préparation des échantillons.}

LES MATÉRIAUX. - Les matériaux utilisés sont de qualité commerciale. La céramique est l'alumine polycristalline Al23 de Degussa de pureté 99,7 \% (cf. Tab. III). Les impuretés présentes sont principalement ségrégées aux joints de grain. Les bases des échantillons cylindriques sont rectifiées, rodées puis polies à la pâte diamantée de 15 à $1 \mu \mathrm{m}$ afin d'obtenir un poli miroir. L'état de surface est alors caractérisé par un coefficient de rugosité $R_{\mathrm{a}}=0,02 \mu \mathrm{m}$.

Tableau III. - Impuretés présentes dans l'alumine.

[Impurities into alumina.]

$\begin{array}{cr}\mathrm{MgO} & 3000 \mathrm{ppm} \\ \mathrm{CaO} & 500 \mathrm{ppm} \\ \mathrm{Na}_{2} \mathrm{O} & 1000 \mathrm{ppm} \\ \mathrm{Fe}_{2} \mathrm{O}_{3} & 400 \mathrm{ppm} \\ \mathrm{SiO}_{2} & 1000 \mathrm{ppm}\end{array}$

Le cuivre OFHC (faible teneur en oxygène) polycristallin est laminé en feuille de $0,2 \mathrm{~mm}$ d'épaissseur. Il est alors restauré par un recuit de $30 \mathrm{mn}$ à $1000^{\circ} \mathrm{C}$ sous vide primaire $\left(10^{-3}\right.$ torr). Des pastilles sont découpées au diamètre de la céramique et polies à la pâte diamantée de $3 \mu \mathrm{m}$. L'état de surface du cuivre avant la liaison est donné par $R_{\mathrm{a}}=0,12 \mu \mathrm{m}$.

\section{ELABORATION DES COUPLES.}

Thermocompression. - Les couples assemblés par thermocompression se présentent sous forme d'éprouvettes "sandwich" dont les extrémités sont constituées par deux cylindres d'alumine entre lesquels sont disposées en alternance une ou plusieurs pastilles de cuivre et d'alumine de 0,2 $\mathrm{mm}$ d'épaisseur. 
La thermocompression $[16,41,43]$ est réalisée dans les conditions suivantes :

- la température est de $100^{\circ} \mathrm{C}$

- la vitesse de montée en température du four est de $5^{\circ} \mathrm{C} / \mathrm{mn}$

- la pression appliquée de façon uniaxiale est comprise entre 1 et $8 \mathrm{MPa}$

- la durée du traitement varie de $30 \mathrm{mn}$ à 6 heures

- l'atmosphère est neutre (argon). La pression totale est d'une atmosphère, la pression partielle d'oxygène étant de l'ordre de $10^{-3}$ torr.

L'apport d'oxygène au niveau de l'interface a été fait sous forme d'une oxydation préalable du cuivre dans une atmosphère à pression d'oxygène controlée de $10^{-1}$ torr et à $1000^{\circ} \mathrm{C}$. L'épaisseur de la couche d'oxyde a été mesurée par décharge luminescente après étalonnage [41]. L'oxyde $\mathrm{Cu}_{2} \mathrm{O}$ (cuprite) a été préféré à l'oxyde $\mathrm{CuO}$ parce qu'il reste adhérent sur son support métallique. Son épaisseur initiale influe sur la tenue mécanique de l'ensemble. Les mesures de résistance au cisaillement effectuées par Courbière (Fig. 4) montrent qu'une épaisseur de 0,7 $\mu \mathrm{m}$ semble optimale.

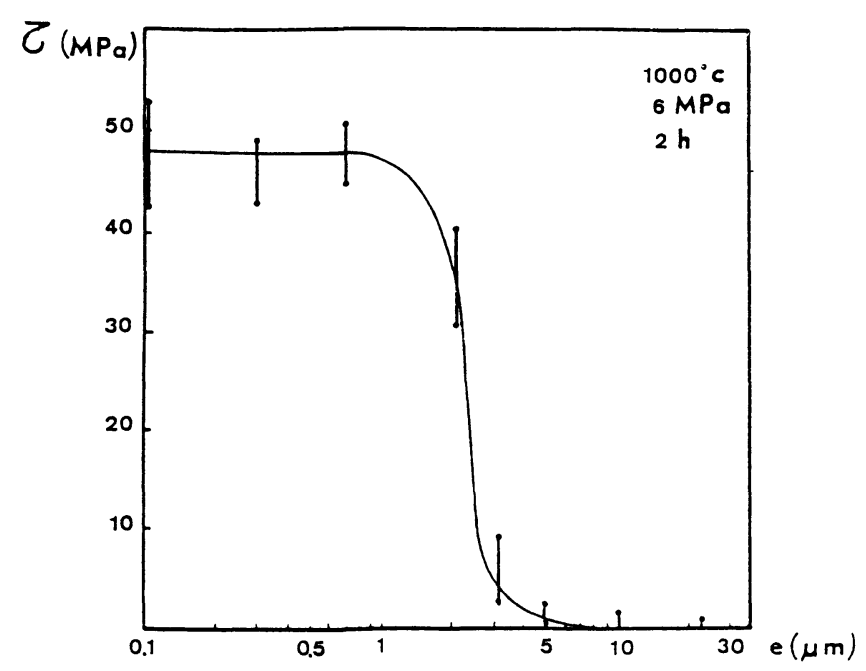

Fig. 4. - Variation de la contrainte à rupture en fonction de l'épaisseur initiale de $\mathrm{Cu}_{2} \mathrm{O}$ [41].

[Dependence of the breaking strength as a function of the $\mathrm{Cu}_{2} \mathrm{O}$ initial thickness [41].]

Eutectique gaz-métal. - L'idée qui prévaut à l'utilisation de cette technique, dite de l'eutectique gaz-métal, est la suivante : le système $\mathrm{Cu}-\mathrm{O}$ (Fig. 5) présente un point eutectique pour une composition en poids de $0,39 \%$ d'oxygène (1,54\% at). La température de fusion de l'eutectique est de $1065^{\circ} \mathrm{C}$ soit $18{ }^{\circ} \mathrm{C}$ en dessous de la température de fusion du cuivre. Les feuilles de cuivre préalablement oxydées sous forme $\mathrm{Cu}_{2} \mathrm{O}$ d'épaisseur variable ainsi que l'alumine sont portées à une température de $1070{ }^{\circ} \mathrm{C}$ dans un four balayé par un flux d'argon. A cette température, une phase liquide de composition proche de l'eutectique se forme d'abord en surface du cuivre puis vient mouiller entièrement la surface de l'alumine. Les figures $6 \mathrm{a}$ et $6 \mathrm{~b}$ montrent les étapes du développement de l'eutectique : en a) premier stade de la formation de l'eutectique localisé à l'interface $\mathrm{Cu} / \mathrm{Cu}_{2} \mathrm{O}$. En b), la diffusion rapide de l'oxygène vers le cuivre massif permet à l'eutectique liquide de venir au contact de la céramique. On notera sur cette figure, l'effet du joint de grain sur l'augmentation de la diffusion. 


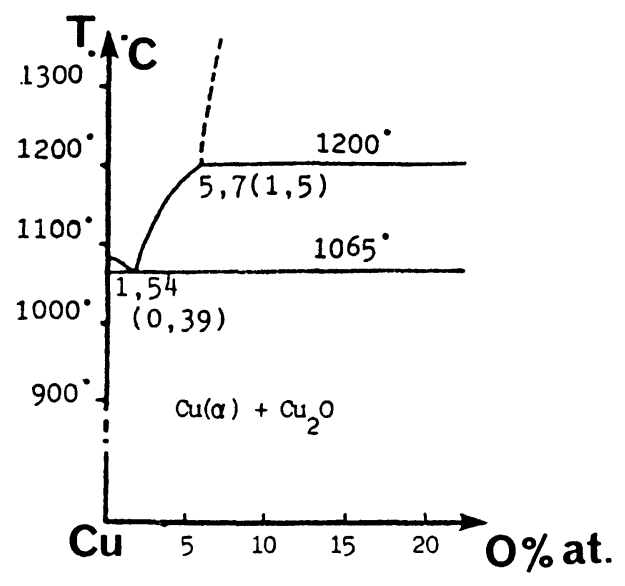

Fig. 5. - Diagramme Cu-O d'après Hansen [42].

[Cu-O diagram proposed by Hansen [42].]

a

\section{Eutectique}

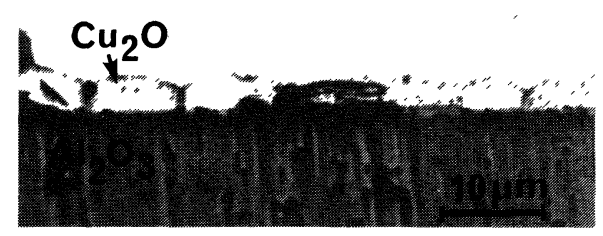

b

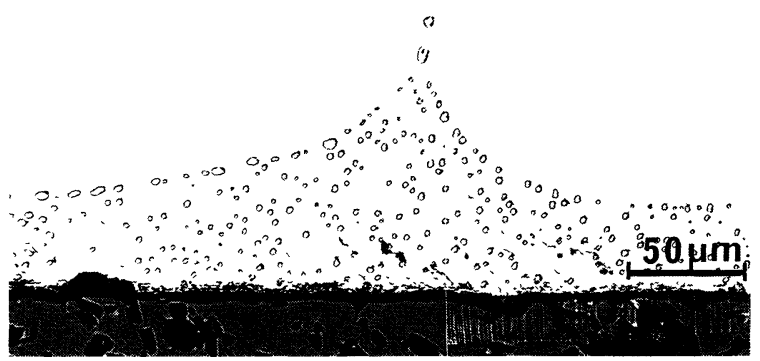

Fig. 6. - a,b) Infiltration du liquide à l'interface obtenue par liaison eutectique. Micrographies optiques réalisées par Courbière [41].

[a, $b$ - Liquid at the interface obtained by the eutectic method. Courtesy of M. Courbière [41].]

Même en l'absence de pression quelques minutes suffisent à établir le contact entre les deux antagonistes. Cependant là aussi, la tenue mécanique du couple est liée à l'épaisseur initiale de l'oxyde de cuivre. Les mesures de la résistance à la traction montrent que l'épaisseur optimale de $\mathrm{Cu}_{2} \mathrm{O}$ est d'environ $7 \mu \mathrm{m}[41,43]$.

PRÉPARATION DES LAMES MINCES. - L'étude au microscope électronique en transmission de l'interface métal/céramique est réalisée principalement grâce à l'examen de coupes transverses (interface parallèle au faisceau). La préparation des lames minces est délicate puisqu'il faut amincir simultanément des matériaux aux caractéristiques très éloignées. Les éprouvettes sont tronçonnées longitudinalement sous forme de plaquettes. Une pastille de $3 \mathrm{~mm}$ de diamètre est carottée dans la zone centrale à l'aide d'un trépan diamanté (Fig. 7). Elle est ensuite préamincie 
mécaniquement à l'aide d'un dispositif à meulage concave (Dimple grinder Gatan) qui permet d'amener la zone centrale de la pastille à une épaisseur de 10-20 $\mu \mathrm{m}$.

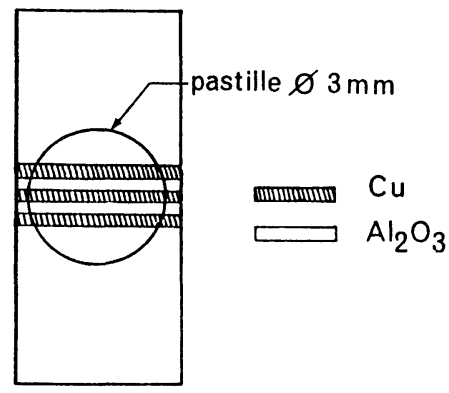

Fig. 7. - Découpe des échantillons.

[Drilling of samples.]

L'amincissement final est réalisé par bombardement ionique à canons d'argon $(5 \mathrm{kV}, 1 \mathrm{~mA}$, $15^{\circ}$ d'inclinaison). Une faible épaisseur au centre de la pastille est indispensable car elle évite que le cuivre ne disparaisse complètement avant que l'alumine ne soit amincie, et tout ceci en raison de l'écart important entre les vitesses d'abrasion du métal et de la céramique. Les observations sont ensuite réalisées au microscope électronique à transmission Jeol 200CX Side Entry (Centre d'Etudes et de Caractérisations Microstructurales de l'INSA de Lyon) équipé d'un jeu de pièces polaires SHP $\left( \pm 25^{\circ}\right)$ et $\operatorname{SAP}\left( \pm 60^{\circ}\right)$.

\section{Résultats expérimentaux.}

LIAISON OBTENUE PAR THERMOCOMPRESSION. - Les résultats des essais en cisaillement $[41,43]$ indiquent que le paramètre pression n'est plus aussi déterminant dès lors qu'elle atteint un niveau d'environ $4 \mathrm{MPa}$. Quant aux paramètres température et temps, ils sont évidemment liés puisque les mécanismes qui favorisent l'adhésion sont thermiquement activés [44] ; on peut invoquer les mécanismes de fluage de la composante métallique, de diffusion superficielle et volumique, d'évaporation-condensation dans les cavités de l'interface.

Nous avons choisi d'étudier l'influence du temps pour une température de $1000^{\circ} \mathrm{C}$ en conservant la même épaisseur initiale de l'oxyde cuivreux en surface du cuivre, soit $0,7 \mu \mathrm{m}$.

Temps "court" : 2 heures . - L'observation par la tranche de l'interface en MET révèle la présence d'un composé interfacial identifié par diffraction à l'oxyde mixte $\mathrm{CuAlO}_{2}($ Fig. 8). Par ailleurs, cet oxyde est facilement reconnaissable grâce à ses macles de croissance. Une étude cristallographique et microstructurale de cet oxyde trigonal (décrit en axes hexagonaux) est présentée à la fin de ce paragraphe, où nous montrons que ces défauts plan s'étendent dans les plans de base de la structure. Son épaisseur varie de 0,2 à $0,4 \mu \mathrm{m}$ et il n'existe aucune relation d'orientation particulière entre les trois matériaux. On remarque que les macles de croissance présentes dans les grains d'oxyde mixte sont parallèles à l'interface ; elles indiquent que la direction de l'axe c est perpendiculaire à l'interface. Nous avons là un résultat remarquable sur la disposition du composé intermédiaire, son plan basal coïncide systématiquement avec le plan de l'interface.

L'aspect très perturbé de la surface du cuivre résulte d'une part d'un effet de dégradation induite par le bombardement ionique et d'autre part, de la présence de fins précipités. Des taches 

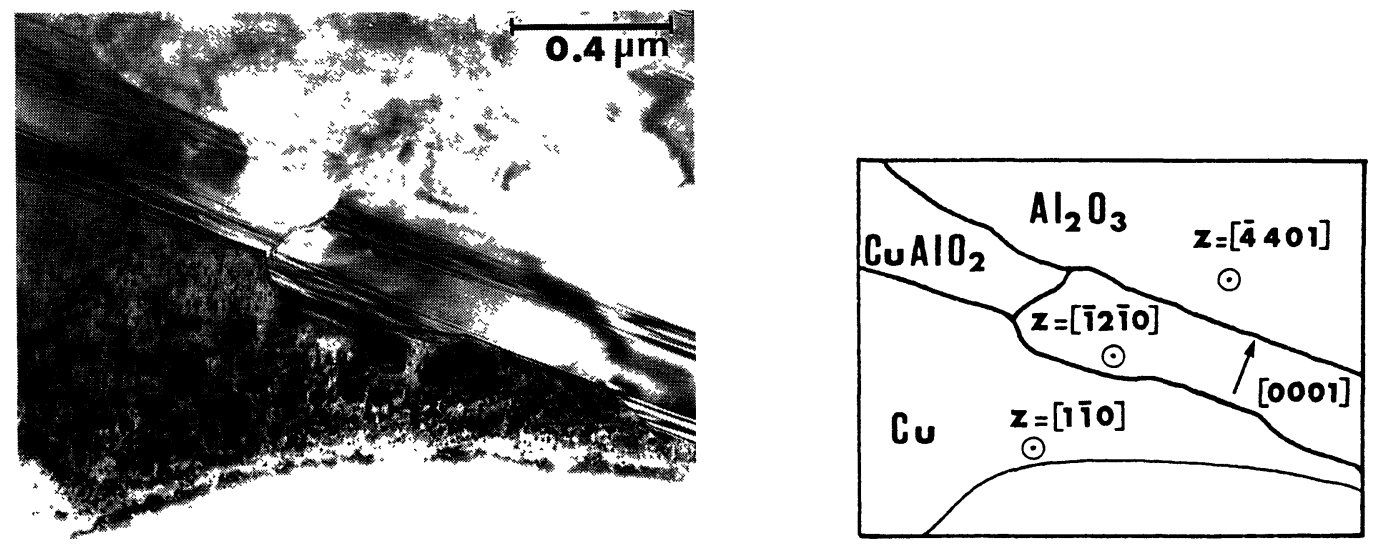

Fig. 8. - Composé à l'interface obtenue par thermocompression $\left(2 \mathrm{~h}\right.$ à $\left.1000^{\circ} \mathrm{C}\right)$.

[Reaction layer at the interface obtained by hot-pressing $\left(2 \mathrm{~h}\right.$ at $\left.1000^{\circ} \mathrm{C}\right)$.]

de diffraction de faible intensité sur les diagrammes du cuivre révèle que l'oxyde $\mathrm{Cu}_{2} \mathrm{O}$ est présent dans celui-ci et qu'il se trouve en épitaxie avec le réseau du métal (Fig. 9). Les relations observées sont d'ailleurs en accord avec les résultats de la littérature $[45,46]$ :

$(001)_{\mathrm{Cu}} / /(001)_{\mathrm{Cu}_{2} \mathrm{O}}$ avec $[1 \overline{1} 0]_{\mathrm{Cu}} / /[1 \overline{1} 0]_{\mathrm{Cu}_{2} \mathrm{O}}$ (épitaxie parfaite)
$(001)_{\mathrm{Cu}} / /(111)_{\mathrm{Cu}_{2} \mathrm{O}}$ avec $[1 \overline{1} 0]_{\mathrm{Cu}} / /[1 \overline{1} 0]_{\mathrm{Cu}_{2} \mathrm{O}}$

Le cuivre présente également des dislocations au voisinage de l'interface, résultat du mécanisme de fluage.

L'alumine située en bordure de l'oxyde mixte ne présente que de rares dislocations sans que l'on puisse les attribuer à un effet de préparation de la céramique plutôt qu'aux contraintes résiduelles de l'assemblage.

Certaines observations effectuées en vue perpendiculaire à l'interface sur le même type d'échantillons après rupture en cisaillement, indiquent que l'oxyde mixte se répartit sous forme de longues aiguilles qui s'enchevêtrent [15].

Temps "long" : 6 heures. - Dans ce cas, la couche intermédiaire d'oxyde mixte a disparu pour laisser place à une couche d'alumine (Fig. 10). Son épaisseur est voisine de $0.2 \mu \mathrm{m}$ et il n'existe pas de relation d'orientation particulière entre l'alumine à l'interface et l'alumine de la matrice.

L'oxyde de cuivre $\mathrm{Cu}_{2} \mathrm{O}$ est toujours présent sous forme de précipités finement dispersés dans la matrice cuivre et en relation d'épitaxie parfaite avec le cuivre.

LIAISON OBTENUE PAR FUSION DE L'EUTECTIQUE. - Les observations menées sur ce type de couple montrent que l'état de la liaison est très proche de celui précédemment détaillé dans le cas de la thermocompression pour le temps de $2 \mathrm{~h}$. Les images en champ sombre de la figure 11 permettent de visualiser chaque composé dont l'oxyde intermédiaire $\mathrm{CuAlO}_{2}$ d'épaisseur moyenne $0.1 \mu \mathrm{m}$. Aucune relation particulière d'orientation n'existe entre les trois matériaux; toutefois l'oxyde mixte contient ici encore des macles de croissance parallèles à l'interface. Certains grains d'oxyde possèdent cependant une morphologie équiaxe, leur taille est comprise entre $0.3 \mathrm{et} 0.5 \mu \mathrm{m}$ sans orientation cristallographique particulière.

La présence de l'oxyde $\mathrm{Cu}_{2} \mathrm{O}$ dans le cuivre au voisinage de l'interface a également été révélée, l'orientation de ses grains est semblable à celle déjà donnée. 

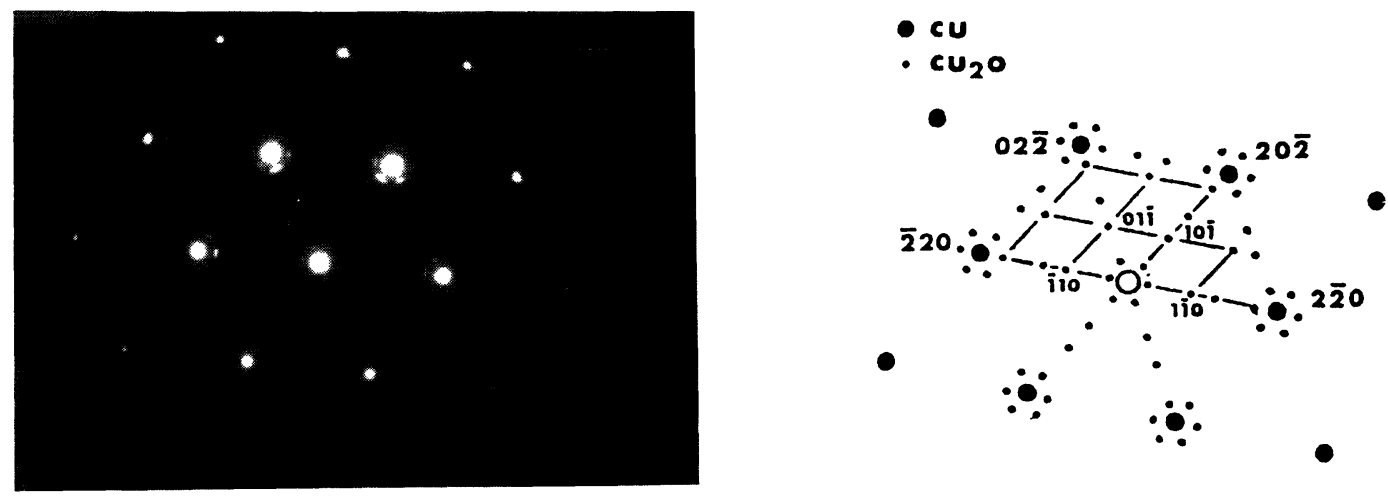

Fig. 9. - Diagramme de diffraction du cuivre. Les taches satellites révèlent la présence de cuprite en épitaxie parfaite avec le réseau du cuivre. Les taches suplémentaires résultent du phénomène de double diffraction.

[Diffraction pattern of copper region. The extra reflections indicate the existence of epitaxial cuprite. The extra spots are due to double diffraction.]
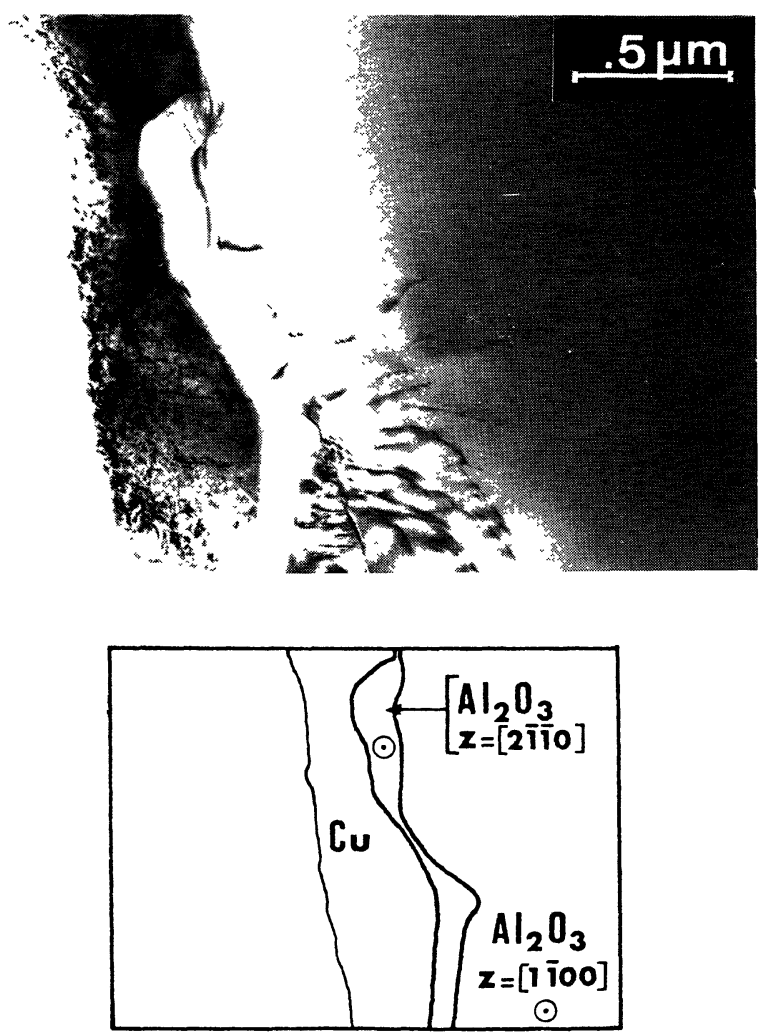

Fig. 10. - Alumine interfaciale après thermocompression $\left(6 \mathrm{~h}-1000^{\circ} \mathrm{C}\right)$.

[Alumina in the interface region for a specimen obtained by hot-pressing $\left(6 \mathrm{~h}\right.$ at $\left.1000^{\circ} \mathrm{C}\right)$.] 


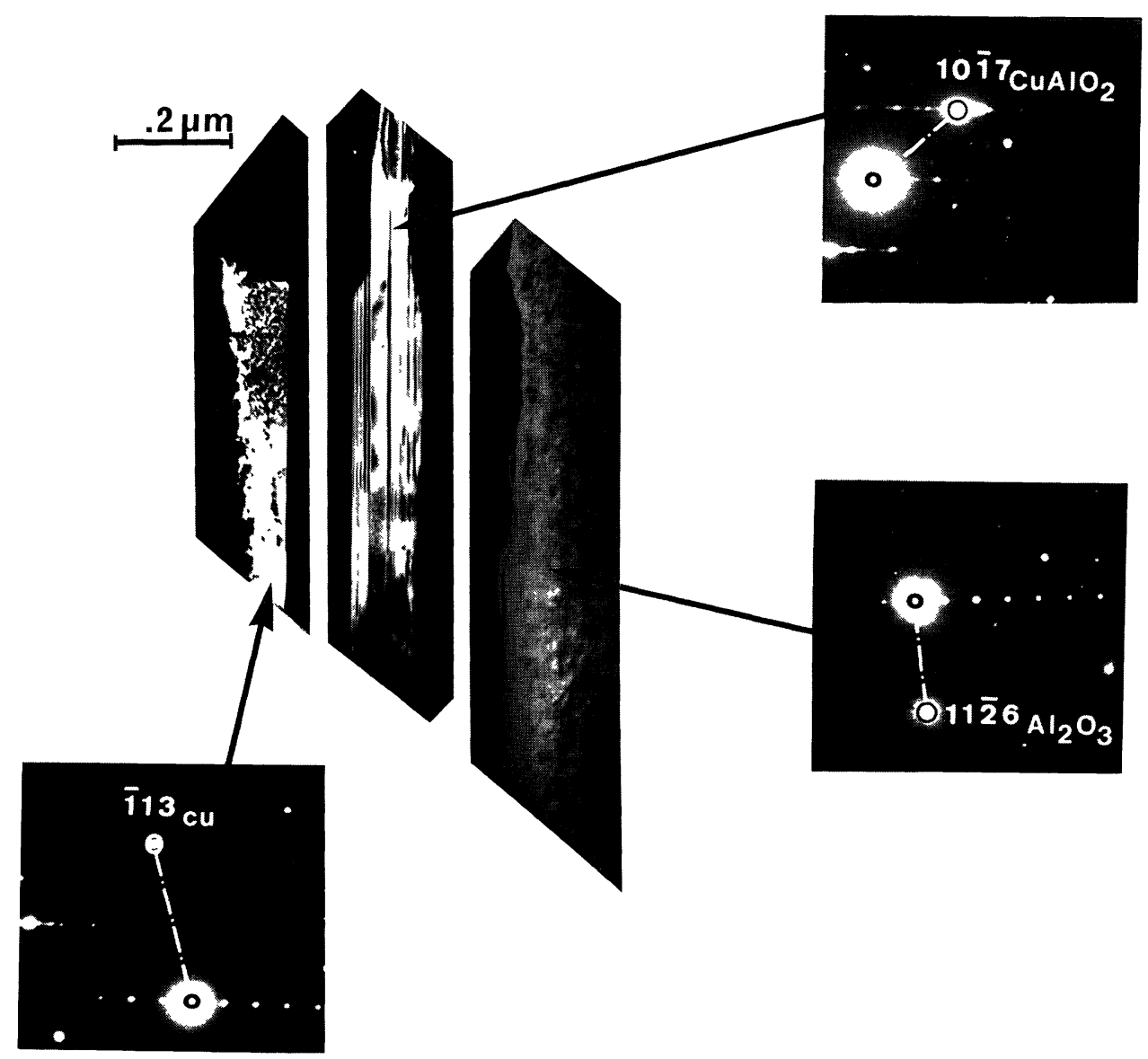

Fig. 11. - Images en champ sombre des constituants présents aux interfaces (liaison par la méthode de l'eutectique).

[Dark field images in the region of the $\mathrm{Al}_{2} \mathrm{O}_{3} / \mathrm{Cu}$ boundary (eutectic method).]

CARACTÉRISATION DE L'OXYDE MIXTE $\mathrm{CuAlO}_{2}$. - Afin d'isoler cet oxyde, nous avons utilisé la technique dite du "slurry coating" qui permet d'obtenir de manière simple des produits de réaction entre une atmosphère, le métal et la céramique. Elle consiste à enduire la surface de l'alumine d'un dépôt de poudre de cuivre en suspension dans un solvant organique et de porter l'ensemble à $1000^{\circ} \mathrm{C}$ dans l'air pendant 100 heures.

L'oxyde de surface observé est le résultat de l'oxydation du cuivre à l'état $\mathrm{Cu}_{2} \mathrm{O}$ qui entre alors en réaction avec l'alumine pour former l'oxyde mixte conformément à la réaction additive :

$$
\mathrm{Cu}_{2} \mathrm{O}+\mathrm{Al}_{2} \mathrm{O}_{3} \longrightarrow 2 \mathrm{CuAlO}_{2}
$$

et au diagramme d'équilibre présenté par la figure 12. Les lames minces sont réalisées en prélevant une tranche de surface qui sera ensuite polie mécaniquement puis ioniquement du côté céramique massive.

L'oxyde mixte $\mathrm{CuAlO}_{2}$ possède une structure Delafossite [47] du type $\mathrm{CsICl}_{2}$. Sa structure 


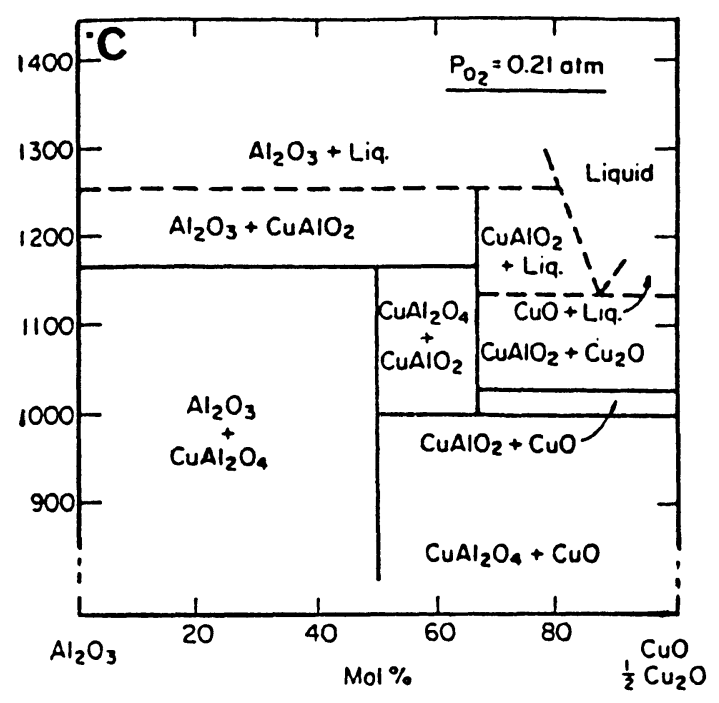

Fig. 12. - Diagramme $\mathrm{Al}_{2} \mathrm{O}_{3}-\mathrm{Cu}_{2} \mathrm{O}[48]$.

$\left[\mathrm{Al}_{2} \mathrm{O}_{3}-\mathrm{Cu}_{2} \mathrm{O}\right.$ diagram [48].]

trigonale, décrite par le groupe d'espace $\mathrm{R} \overline{3} \mathrm{~m}$, possède les paramètres : $a=0,285 \mathrm{~nm}$ et $c=$ $1,698 \mathrm{~nm}$. Les atomes de cuivre occupent la position 3a de Wyckoff ; les atomes d'aluminium occupent la position $3 b$ et les atomes d'oxygènes la position $6 \mathrm{c}(z=0,1098)$. Une image de microscopie à haute résolution de cette structure vue selon [1120] est montrée par la figure 13a..

Les conditions de travail, détaillées dans la légende, indiquent que les colonnes atomiques apparaissent en blanc. La simulation de l'image par la méthode des ondes de Bloch à l'aide du logiciel EMS [49] implanté au laboratoire nous enseigne que les points les plus intenses représentent les atomes de cuivre, les autres étant associées aux groupements $\mathrm{Al}-\mathrm{O}$. La maille de l'oxyde se présente donc un empilement des couches alternées de groupements $\mathrm{AlO}_{2}$ et $\mathrm{Cu}$, parallèlement à l'axe $c$ (Fig. 14). Les ions cuivre sont coordonnés linéairement à deux ions oxygène pour former les groupements $\mathrm{CuO}_{2}$ déjà présents dans l'oxyde $\mathrm{Cu}_{2} \mathrm{O}$. Les ions $\mathrm{Al}^{3+}$ sont liés à 6 ions $\mathrm{O}^{2-}$ et forment ainsi des octaèdres $\mathrm{AlO}_{6}$ que l'on rencontre dans l'alumine [46]. La cristallographie de l'oxyde mixte $\mathrm{CuAlO}_{2}$ est donc intermédiaire entre celle de l'alumine et de la cuprite. Elle laisse entrevoir des propriétés physiques très anisotropes comme l'illustrent les valeurs du coefficient de dilatation donnés par le tableau IV.

Tableau IV. - Coefficients de dilatation thermique de $\mathrm{CuAlO}_{2}$ [50], alumine, cuprite et cuivre. [Thermal expansion coefficients of $\mathrm{CuAlO}_{2}$ [50], alumina, cuprite and copper.]

\begin{tabular}{|c|c|c|c|c|}
\hline$\alpha \times 10^{6} \mathrm{~K}^{-1}$ & $\mathrm{CuAlO}_{2}$ & $\mathrm{Al}_{2} \mathrm{O}_{3}$ & $\mathrm{Cu}_{2} \mathrm{O}$ & $\mathrm{Cu}$ \\
\hline selon axe $a$ & 11 & 10 & 1,9 & 17 \\
selon axe $c$ & 4,1 & 11,2 & - & - \\
\hline
\end{tabular}



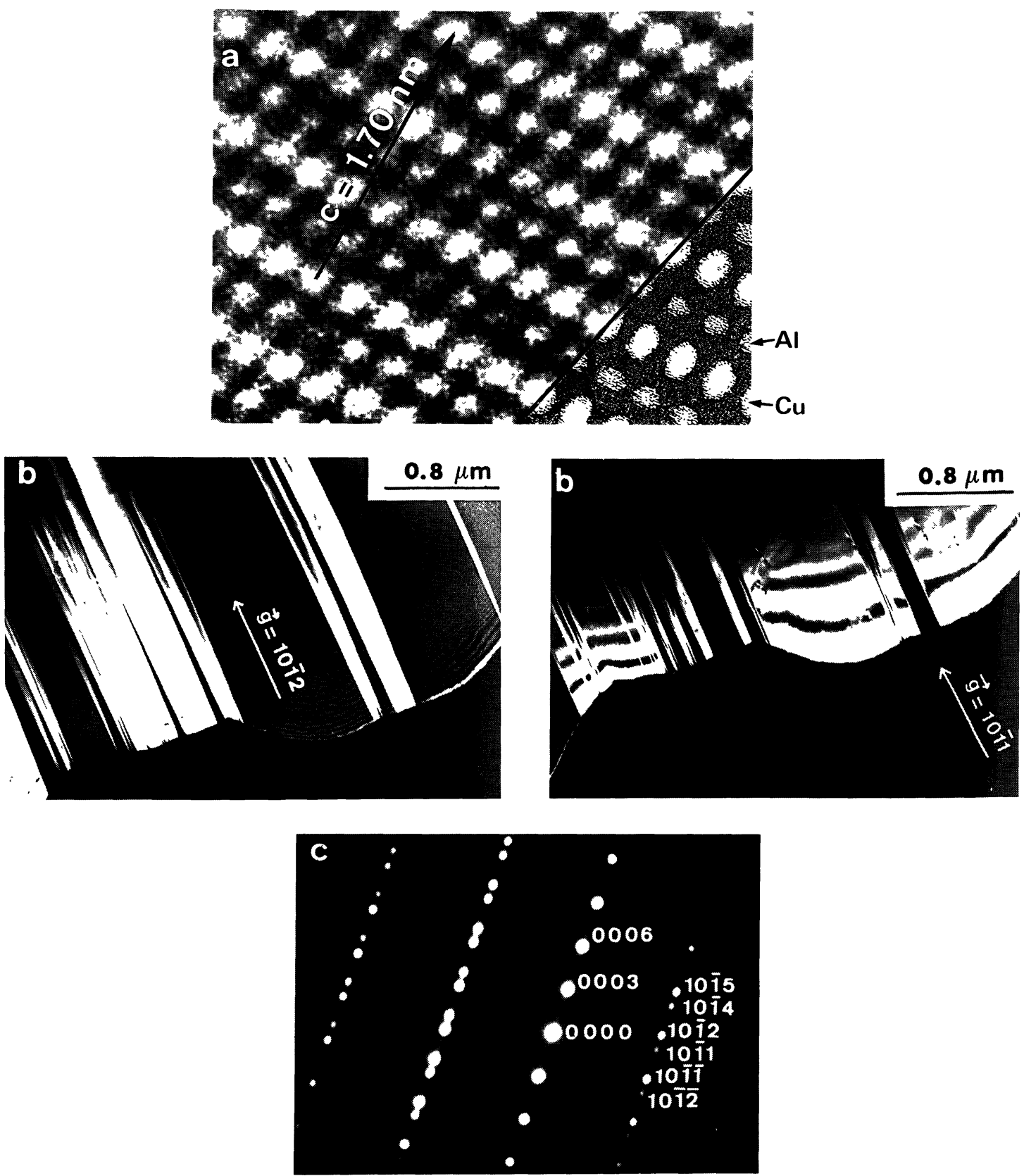

Fig. 13. - a) Image de haute résolution de l'oxyde $\mathrm{CuAlO}_{2}$ en vue [1120] (microscope $300 \mathrm{kV}$, EM $430 \mathrm{ST}$ ) dont la défocalisation de Scherzer est voisine de $-49 \mathrm{~nm}$ ). La simulation juxtaposée a été obtenue à partir du formalisme des ondes de Bloch (61 faisceaux sans absorption, épaisseur $3,4 \mathrm{~nm}$, défocalisation - $65 \mathrm{~nm}$ ). b) Champs sombres de la même zone du cristal. c) Diagramme de la zone (noter la présence de taches régies par $-h+k+l \neq 3 n)$.

[a) High resolution image of $\mathrm{CuAlO}_{2}$ in view along [1120] (300 kV-EM 430 ST microscope having a Scherzer defocus close of $-49 \mathrm{~nm}$ ). The simulation placed side by side has been obtained from the Bloch waves formalism (61 beams without absorption, thickness $3.4 \mathrm{nmn}$, defocus $-65 \mathrm{~nm})$. b) Dark fields of the same region. c) Diffraction pattern of the previous region (notice the presence of spots given by $-h+k+l \neq 3 n$ ).] 


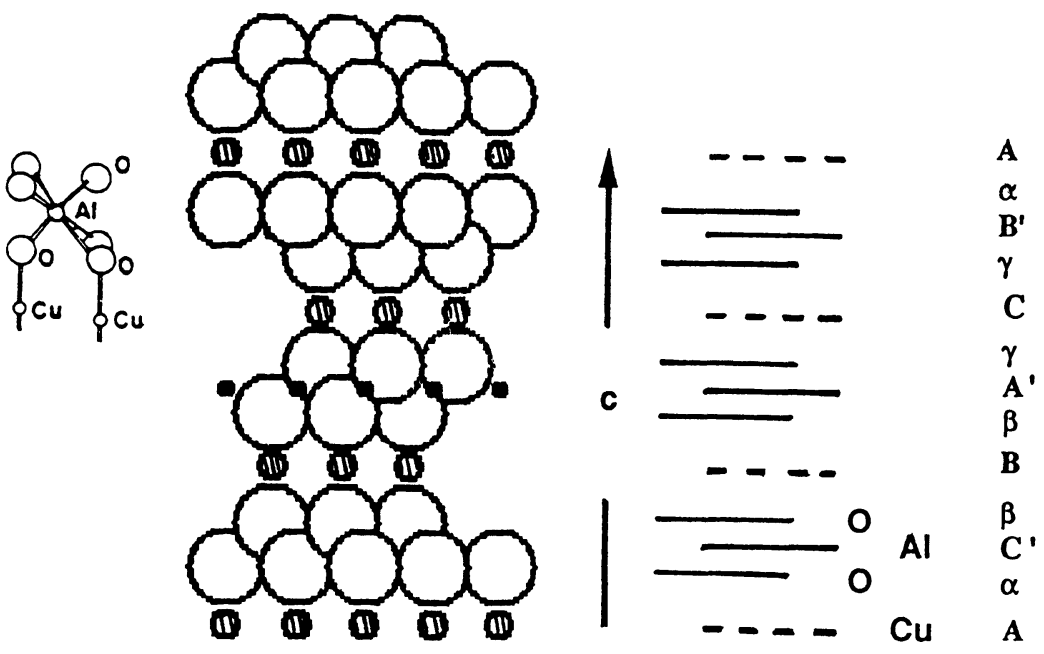

Fig. 14. - Cristallographie de l'oxyde mixte $\mathrm{CuAlO}_{2}$.

[Crystallography of the $\mathrm{CuAlO}_{2}$ binary oxide.]

La figure $13 b$ présente une vue générale de ce matériau. Un contraste en bandes alternativement claires et sombres est observé dans tous les grains de cet oxyde. Les conditions de diffraction régies par le groupe d'espace sont $\bar{h}+k+l=3 n$ signifiant l'extinction de certaines ondes telles que $0001,0002,1 \overline{1} 00,1 \overline{1} 01,1 \overline{1} 02,1 \overline{1} 03,1 \overline{1} 0 \overline{3}$ par exemple.

Sur le diagramme de diffraction de la figure 13c, réalisé à cheval sur deux bandes, apparaissent des taches normalement interdites. Il ressort de son indexation que les deux cristaux possèdent la même orientation cristallographique, seule la nature des atomes dans les sites est différente. Les ondes "supplémentaires" ont l'indexation des ondes normalement présentes à condition de changer $l$ en $-l$, à l'exclusion de $l=3 n$. Les deux cristaux sont donc en position de macle obtenue par retournement de l'axe $c$; ils sont énantiomorphes, le plan de macle coïncidant avec le plan basal (0001). Le grand nombre de macles observées semble indiquer que ce type de défaut structural possède un basse énergie. Par endroits, la succession des macles tend à s'ordonner créant localement une structure polytype comme l'illustre la figure 15. Les images de plans (0003) distants de $0,566 \mathrm{~nm}$ laissent voir des tranches plus claires correspondant à des "micromacles" réparties de façon pratiquement équidistantes.

En outre, nous avons procédé à la détermination des vecteurs de Burgers et des vecteurs de ligne des dislocations présentes. Leur vecteur de Burgers est toujours de type $a=1 / 3\langle 11 \overline{2} 0\rangle$ tandis que leur caractère est le plus souvent purement vis. L'examen dans une direction transversale à l'axe $c$ (Fig. 16) nous permet de s'assurer que les dislocations se répartissent dans le plan basal, qui est alors le plan de glissement de la structure. Quelques rares dislocations présentent une direction proche de $c$ mais elles sont systématiquement ancrées sur des obstacles et non significatives du glissement. En usant de la technique du champ sombre faiblement excité, les dislocations n'apparaissent pas dissociées tout au moins dans la limite de résolution des images obtenues (de l'ordre de $1,5 \mathrm{~nm}$ ). Il scmblerait qu'elles possèdent une certaine mobilité puisque certaines se déplacent sous le faisceau électronique. Cependant, une étude en température réalisée en chauffant in-situ jusqu'à $400^{\circ} \mathrm{C}$ une lame d'oxyde mixte, ne nous a pas permis d'observer un mouvement généralisé initié par les contraintes d'origine thermique. A priori, leur mobilité est certainement 


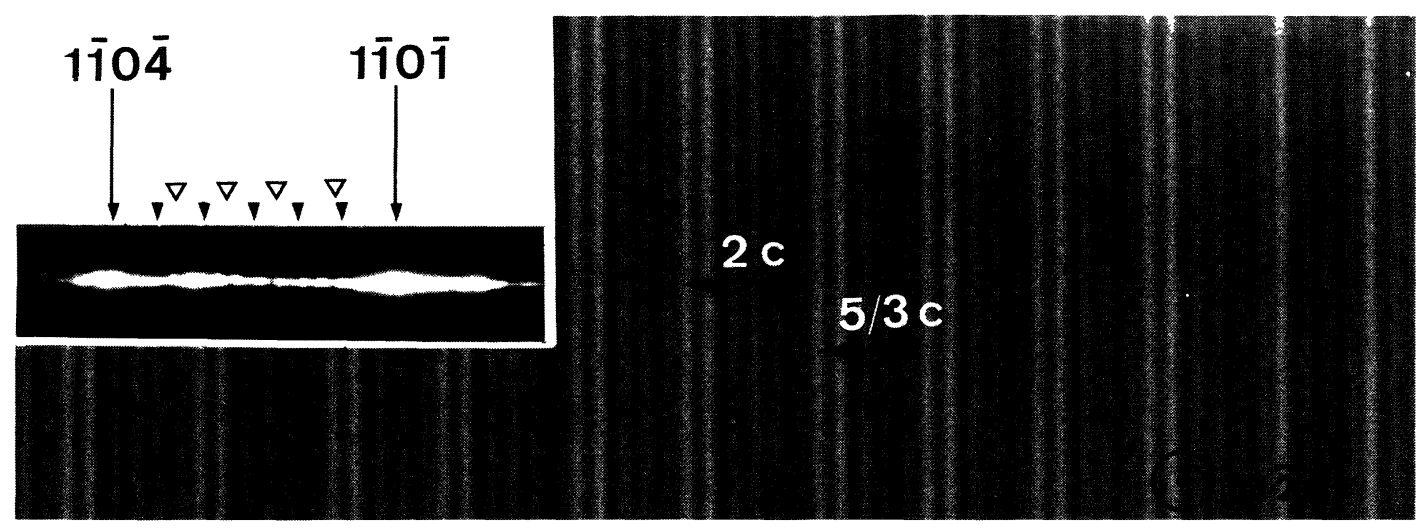

Fig. 15. - Structure "micromaclée" de l'oxyde $\mathrm{CuAlO}_{2}$. La portion du diagramme de diffraction montre les périodes $2 \mathrm{c}(\nabla)$ et $5 / 3 \mathrm{c}(\nabla)$ assez fréquentes.

[Microtwinned structure of $\mathrm{CuAlO}_{2}$ oxide. The partial diffraction pattern shows the most probable periods $2 c(\nabla)$ and $5 / 3 c(\nabla) \cdot]$

plus importante que dans l'alumine comme le laisse supposer l'arrangement atomique de la structure où l'on rencontre des plans entièrement cuivre bien séparés de leur voisins (cf. Fig. 14).

Enfin, le comportement à la rupture de l'oxyde mixte apparaît complexe ; nous avons en effet observé une microfissure se propageant au travers des cristaux maclés (Fig. 17). Celle-ci ne progresse pratiquement pas dans le plan de base mais dans le plan (1101) faisant un angle de 81,7 degrés avec le plan de base. A la traversée d'un cristal énantiomorphe, le plan de la fissure dévie pour conserver la nature chimique de la surface créée. Nous pouvons alors remarquer que les énergies de surface des deux types de plans (1101) et (110ī) sont très différentes, ce qui n'est pas surprenant compte tenu de la nature chimique de ces plans (cf. Fig. 18). Les plans (11101) ne contiennent que les groupements homopolaires $\mathrm{Cu}-\mathrm{O}$ (plans a) ou que les groupements $\mathrm{Al}-\mathrm{O}$ (plans b) tandis que les plans $(1 \overline{1} 0 \overline{1})$ sont plus complexes.

\section{Discussion.}

EVOLUTION DES COMPOSÉS À L'INTERFACE. - Comme le montrent les résultats obtenus par la méthode de fusion de l'eutectique gaz-métal, la réactivité du système $\mathrm{Cu}_{2} \mathrm{O}-\mathrm{Al}_{2} \mathrm{O}_{3}$ vers $1000{ }^{\circ} \mathrm{C}$ est très grande puisque quelques minutes suffisent pour former le produit de réaction $\mathrm{CuAlO}_{2}$. Cependant, des travaux similaires effectués depuis [19], en vue de préparer des couples par la méthode de l'eutectique, semblent indiquer que l'apparition des oxydes mixte et spinelle n'intervient qu'au bout de 6 minutes, avec toutefois, une morphologie très variable d'une plage à l'autre. En effet, outre les oxydes, une phase amorphe intermédiaire est présente, phase déjà observée entre le cuivre et l'alumine pour un palier de 3 minutes au-dessus de l'eutectique.

Dans le cas de la thermocompression, l'établissement de la liaison et le développement des produits de réaction est conditionné par le remplissage des porosités, processus qui, pour être opérant, nécessite des temps de traitement longs. Le processus d'évaporation-condensation du métal à la surface de la céramique accompagné d'une diffusion superficielle des atomes métalliques s'applique de manière satisfaisante aux cas où toute réaction à l'interface est rigoureusement évitée. Les temps de condensation d'une monocouche de cuivre à $1000^{\circ} \mathrm{C}$ a été estimé [4], il est d'une seconde environ. 


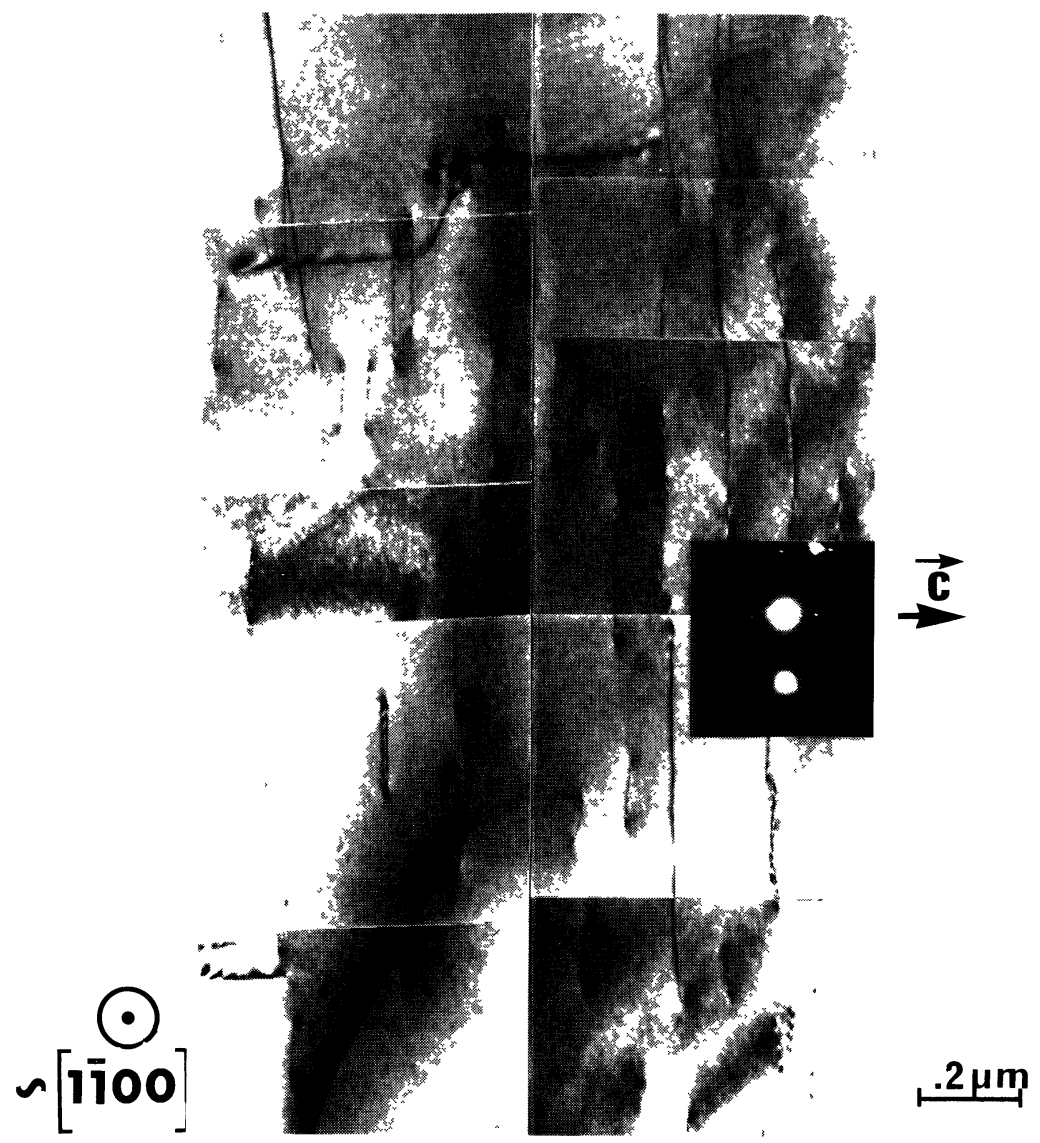

Fig. 16. - Dislocations dans l'oxyde $\mathrm{CuAlO}_{2}$. La plan basal est pratiquement debout. $(g=(11 \overline{2} 0), G=$ 60000).

[Dislocations in $\mathrm{CuAlO}_{2}$ oxide. The basal plane is nearly parallel to the electron beam $(g=(11 \overline{2} 0)$, $G=60000)$.]

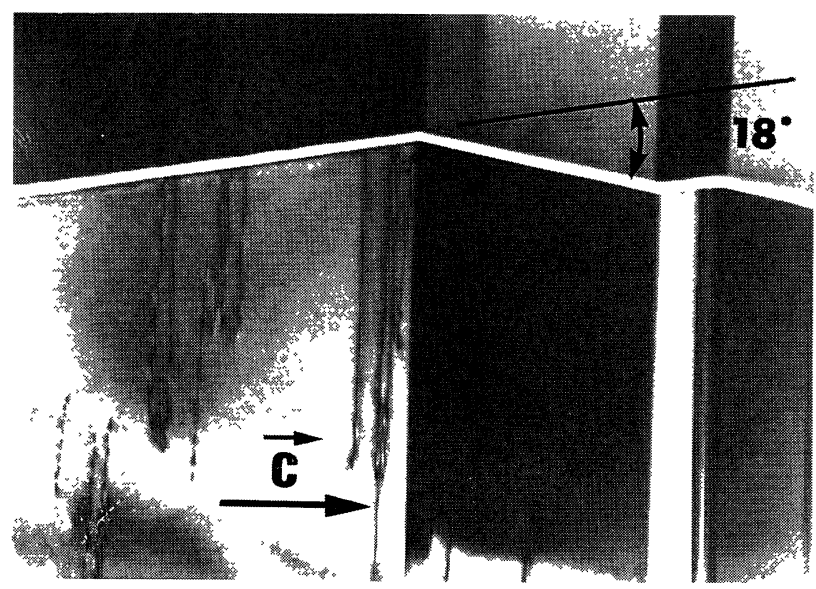

Fig. 17. - Vue $[11 \overline{2} 0]$ d'une région maclée présentant une microfissure.

[Twinned region in view along [1120] ] axis and containing a microcrack.] 


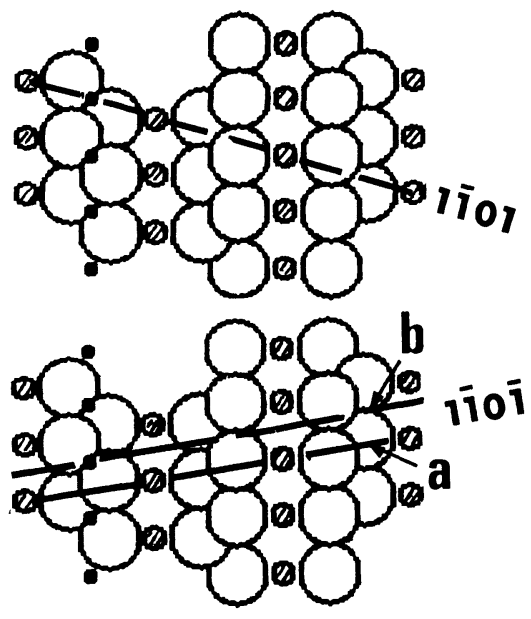

Fig. 18. - Traces des plans (1101) et (11̄0i) .

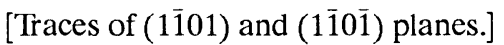

Lorsqu'une réaction entre les constituants a lieu, le phénomène d'interdiffusion conjugué aux aspects de germination-croissance s'ajoute au processus précédent. En fait, le mécanisme de fluage du cuivre possède une grande importance dans l'établissement de la liaison. L'écrasement des aspérités à l'interface est assimilable au processus de fluage type Nabarro-Herring qui donne une vitesse de déformation (taille de grain inférieure au diamètre $d$ des zones en contact) :

$$
\dot{\mathcal{E}}=(A D / d h)\left(\sigma b^{3} / k T\right) \text { soit } \dot{h}=(A D / d)\left(\sigma b^{3} / k T\right)
$$

où $D$ est le coefficient d'autodiffusion, $h$ la hauteur des zones de contact, $A$ un coefficient de l'ordre de 10 [51]. La zone de contact s'accroît au cours du temps mais pour une valeur du diamètre de l'ordre de $100 \mu \mathrm{m}$ à $10 \mathrm{MPa}$, il vient $\dot{h}$ de l'ordre de $0,7 \mu \mathrm{m} / \mathrm{h}$. Le fluage semble prépondérant au début de la thermocompression alors que le processus de diffusion superficielle et d'évaporation deviennent plus efficaces au cours du temps, les cavités restantes présentant localement de faibles rayons de courbure.

Nos observations montrent que la nature des composés interfaciaux diffère suivant la durée du traitement de thermocompression et par voie de conséquence suivant l'état d'oxydation du métal. Après deux heures de traitement à $1000^{\circ} \mathrm{C}$, une couche d'oxyde mixte est observée à l'interface ; elle est remplacée après 6 heures par une couche d'alumine. La succession de l'épaisseur des couches est approximativement la suivante :

$$
\mathrm{Cu}_{2} \mathrm{O}(0,7 \mu \mathrm{m})-2 \mathrm{~h} \longrightarrow \mathrm{CuAlO}_{2}(0,2 \mu \mathrm{m})-6 \mathrm{~h} \longrightarrow \mathrm{Al}_{2} \mathrm{O}_{3}(0,1 \mu \mathrm{m})
$$

Il est raisonnable de considérer que la formation de l'oxyde mixte résulte de la décomposition de la couche superficielle de cuprite au contact de l'alumine selon la réaction globale (4).

En réalité, cette réaction globale n'est pas si aisée à analyser qu'il y paraît. Tout d'abord, elle prévoit une épaisseur de la couche d'oxyde mixte pratiquement double de celle de la cuprite initiale. Or rien de tel n'est observé, la couche de $\mathrm{Cu}_{2} \mathrm{O}$ s'amenuise donc pendant le temps de la réaction. Ensuite, la croissance de l'oxyde mixte impose la diffusion des cations et peut-être des anions oxygène. En fait une étude comparative des valeurs du coefficient de diffusion dans 

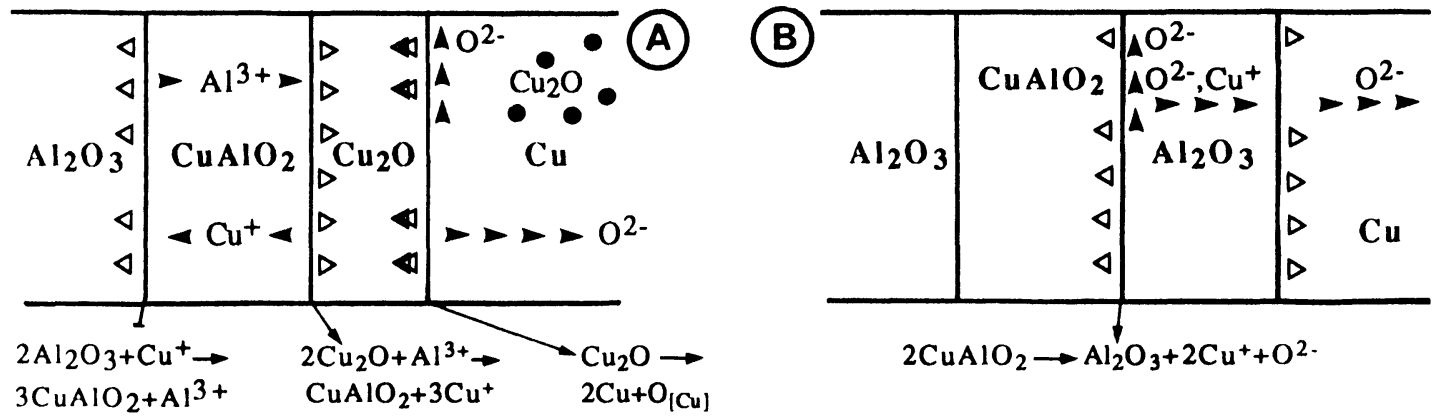

Fig. 19. - Schémas des échanges interfaciaux.

[Schematic interchange in the interface region.]

Tableau V. - Coefficients de diffusion de certaines espèces dans l'alumine, la cuprite et le cuivre. [Diffusion coefficients of different species in alumina, cuprite and copper.]

\begin{tabular}{|cc|c|c|c|c|}
\hline \multicolumn{2}{|c|}{ Matrice } & Espèce & $\begin{array}{c}Q \text { en } \\
\mathrm{kcal} / \mathrm{mole}\end{array}$ & $\begin{array}{c}D \text { à } 1000^{\circ} \mathrm{C} \\
\text { en } \mathrm{cm}^{2} / \mathrm{s}\end{array}$ & Ref. \\
\hline & poly & $\mathrm{Al}$ & 114 & $8 \times 10^{-19}$ & {$[53]$} \\
& mono & $\mathrm{Fe}$ & 71,8 & $1,8 \times 10^{-17}$ & {$[54]$} \\
$\mathrm{Al}_{2} \mathrm{O}_{3}$ & $\prime \prime$ & $\mathrm{Cr}$ & 63,6 & $8,4 \times 10^{-18}$ & {$[54]$} \\
& $\prime \prime$ & $\mathrm{Ni}$ & 67 & $8,2 \times 10^{-18}$ & {$[55]$} \\
& $\prime \prime$ & $\mathrm{Ag}$ & 79 & $7 \times 10^{-14}$ & {$[56]$} \\
& & $\mathrm{O}$ & 152 & $1,6 \times 10^{-23}$ & {$[57]$} \\
$\mathrm{Cu}_{2} \mathrm{O}$ & poly & $\mathrm{Cu}$ & 28,5 & $1,1 \times 10^{-8}$ & {$[58]$} \\
& $\prime \prime$ & $\mathrm{O}$ & 35,7 & $1,4 \times 10^{-10}$ & {$[59]$} \\
& & & & $\left(p \mathrm{O}_{2}=10^{-3}\right.$ atm $)$ & \\
& & & & & \\
$\mathrm{Cu}$ & poly & $\mathrm{Cu}$ & 49,6 & $1,9 \times 10^{-9}$ & {$[60]$} \\
& $\prime \prime$ & $\mathrm{O}$ & 13,7 & $\approx 10^{-5}$ & {$[61]$} \\
\hline
\end{tabular}

certains oxydes (cf. Tab. V) nous pousse à admettre la prépondérance de la diffusion cationique. Le détail de l'évolution des composés interfaciaux est alors résumé sur la figure 19a.

La croissance de l'oxyde mixte résulte de la diffusion corrélée et en sens opposé des cations à travers le composé. Ce processus est analogue à celui intervenant dans la formation de la spinelle $\mathrm{ZnAl}_{2} \mathrm{O}_{4}$ par réaction entre oxydes [52]. La faible épaisseur du composé résulte d'un flux important d'oxygène vers le cuivre massif, permis par la forte valeur du coefficient de diffusion de l'oxygène dans le cuivre (cf. Tab. V). Ceci explique la réduction rapide de l'épaisseur de la cuprite 
qui d'ailleurs se retrouve en partie sous forme de petits nodules dans le cuivre. Il n'est pas exclu que de l'oxygène gazeux se forme à l'interface en formant des bulles comme certains examens du faciès de rupture semblent le laisser supposer [8].

Après l'achèvement de ce processus, l'équilibre thermodynamique n'est pas encore atteint. L'oxyde le plus stable étant l'alumine, on doit s'attendre, en l'absence de la cuprite, à une décomposition de l'oxyde mixte au bénéfice de l'alumine selon un processus détaillé par la figure $19 \mathrm{~b}$. Elle passe par une diffusion des atomes de cuivre au travers de la couche d'alumine naissante. Une partie des ions oxygène libérés peuvent diffuser également et disparaître dans le cuivre mais, certainement, la plupart diffusent le long de l'interface ou forment des bulles. On remarquera enfin que la réaction de décomposition du composé $\mathrm{CuAlO}_{2}$ prévoit une épaisseur de la couche d'alumine environ moitié de celle de l'oxyde mixte, ce que nous observons. D'autre part, l'alumine naissante ne peut se développer qu'à l'interface cuivre/oxyde mixte expliquant ainsi l'absence de relation cristallographique particulière entre les deux réseaux d'alumine.

ORIENTATION CRISTALLOGRAPHIQUE DE L'OXYDE MIXTE. - Sur la figure 8 et la figure 11 qui montrent le composé interfacial $\mathrm{CuAlO}_{2}$, les macles d'énantiomorphisme sont visibles, indiquant que le plan basal de la structure est toujours orienté parallèlement au plan de l'interface. Cette disposition est adéquate pour une meilleure adaptation des écarts de dilatation entre les deux oxydes comme le rappelle le tableau III. Les cisaillements dans le plan interfacial sont ainsi réduits mais, de plus, il peuvent être adaptés par une déformation plastique du $\mathrm{CuAlO}_{2}$, le plan d'accolement étant aussi le plan de glissement. Cependant, cette plasticité n'est opérante qu'à une température suffisamment élevée mais, semble-t-il, pas par trop. A ce propos, l'absence probable d'une dissociation des dislocations dans cet oxyde n'est pas surprenante. Une éventuelle dissociation de Shockley produirait un cisaillement détruisant la liaison homopolaire $\mathrm{O}-\mathrm{Cu}-\mathrm{O}$ si le plan de dissociation coïncidait avec le plan cuivre ou distordant le groupement octaédrique $\mathrm{AlO}_{6}$ si la dissociation avait lieu dans le plan des atomes d'aluminium (cf. empilement ci-dessous).

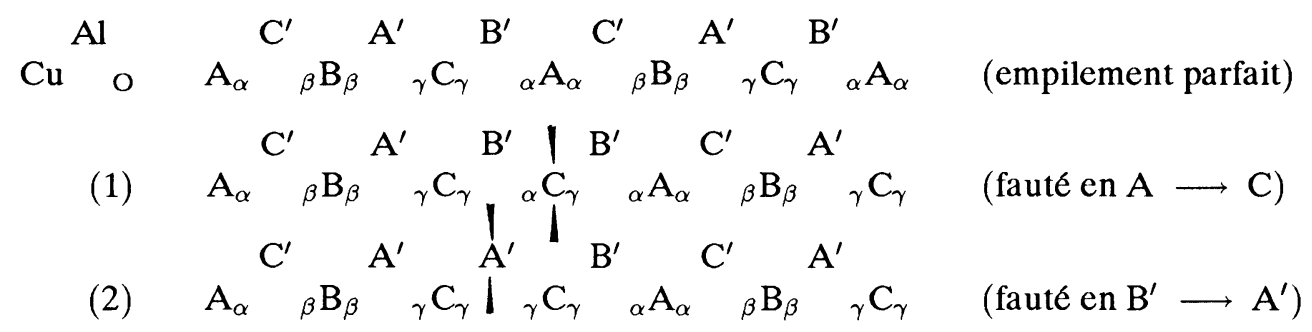

Dans les deux cas, les groupements détruits sont trop stables pour que la dissociation soit favorable. Cependant le plan de glissement réel n'est pas connu ; on peut préssentir qu'il coïncide plutôt avec le plan des atomes de cuivre, le cisaillement de la liaison homopolaire semblant plus facile.

Un élément de réponse peut être obtenu par l'analyse fine des macles de cet oxyde. Elles consistent en un renversement de la maille mais en fait, elles peuvent être décrites par une simple rotation de $60^{\circ}$ dans le plan basal. Une étude par microscopie électronique à haute résolution [62] a permis de montrer que le plan des macles coïncide avec un plan cuivre. La succession de l'empilement rappelée ci-dessous :

$$
\mathrm{A}_{\alpha}{ }_{\beta}^{\mathrm{C}^{\prime}} \mathrm{B}_{\beta}{ }_{{ }_{\gamma} \mathrm{C}_{\gamma}}^{\mathrm{A}^{\prime}}{ }_{\alpha} \mathrm{B}_{\alpha}^{\prime}{ }_{\gamma} \mathrm{C}_{\gamma}{ }_{\beta} \mathrm{B}_{\beta}{ }_{\alpha} \mathrm{A}_{\alpha} \quad \text { (maclé en } \mathrm{A} \text { ) }
$$

montre que la liaison homopolaire autour des atomes de cuivre de l'interface est conservée de part et d'autre de l'interface, de même que les groupements octaédriques autour des atomes 
d'aluminium. Quant aux atomes métalliques, ils occupent toujours les sites octaédriques mais ils sont juste inversés deux plans sur trois. Cette inversion se produit au moment de la croissance de l'oxyde qui semble donc se faire dans la direction c orthogonale au plan de l'interface. Ces considérations sur la nature chimique des macles d'énantiomorphisme suggèrent fortement que le plan de glissement de la structure est aussi au plan cuivre. Cette affirmation semble corroborée par des observations préliminaires à haute résolution qui seront développées ultérieurement [63].

\section{Conclusion}

La qualité de la liaison cuivre/alumine est conditionnée par les possibilités réactionnelles de la cuprite avec l'alumine. L'agent déterminant est l'oxygène qui favorise la réaction (ou accroît le mouillage). La tenue mécanique est liée à l'épaisseur, à la disposition et aux caractéristiques physiques et microstructurales du composé interfacial $\mathrm{CuAlO}_{2}$, fruit de la réaction additive entre $\mathrm{Cu}_{2} \mathrm{O}$ et $\mathrm{Al}_{2} \mathrm{O}_{3}$. Ce composé interfacial de symétrie trigonale est systématiquement orienté de sorte à placer le plan principal de sa structure parallèlement au plan de l'interface. Cette disposition est favorable pour l'adaptation des écarts de dilatation entre la céramique et le substrat métallique.

\section{Remerciements.}

Les auteurs remercient Messieurs P. Buffat et P. Stadelmann de l'Institut Interdépartemental de Microscopie de l'Ecole Polytechnique Fédérale de Lausanne pour l'utilisation du microscope EM $430 \mathrm{ST}$ et les nombreuses discussions à propos des images de haute résolution et leurs simulations.

\section{Bibliographie}

[1] Borbidge W. E., Allen R. V. et Whelan P. T., J. Phys. Colloq. France 47 (1986) C1-131

[2] NichOlas M. G., Science of Ceramics 5, publié par le Swedish Institute for Silicate Research, Eds. C. Brosset et C. Knapp (1970) p. 214 ; Fondamentals of diffusion bonding, Ed. Y. Ishida, Studies Phys. Theor. Chem. (Elsevier) 48 (1987) 3.

[3] Nicholas M. G., Valentine T. M., Waite M. J., J. Mater. Sci. 15 (1980) 2197.

[4] KıOMP J. T., Science of Ceramics 5 publie par le Swedish Inst. of Silicate Research, Eds. C. Brosset et C. Knapp (1970) p. 501 ; Ceram. Bull. 51 (1972) 683 ; Fondamentals of diffusion bonding, Ed. Y. Ishida, Studies Phys. Theor. Chem. (Elsevier) 48 (1987) 3.

[5] Nicholas M. G., CRISPIN R. M., J. Mater. Sci. 17 (1982) 3347.

[6] Bermudez V. M., Appl. Phys. Lett. 42 (1983) 70.

[7] De Bruin H. J., MoOdie A. F., Warble C. E., J. Mater. Sci. 7 (1972) 909.

[8] BRUIN H. J., VANDER POORTEN H., Silicates Industriels 10 (1981) 201 ; 11 (1981) 219.

[9] Dalgleish B. J., Lu M. C. and Evans A. G., Acta Met. 36 (1988) 2029.

[10] Allen R. V., Borbidge W. E., J. Mater. Sci. 18 (1983) 2835.

[11] CALOW C. A., BAYER P. D., PORTER I. T., J. Mater. Sci. 6 (1971) 150, 156.

[12] Morozumi S., KikUChi M., Nishino T., J. Mater. Sci. 16 (1981) 2137.

[13] TurwitT M., Mucke H., OPIOlKa S., ElsSNer G., Prakt. Met. 23 (1986) 325.

[14] BURGER K., MADER W., RHULE M., Ultramicroscopy 22 (1987) 1.

[15] BERAUD C., Thèse de l'Université Lyon (1987).

[16] Courbiere M., Treheux D., Beraud C., Esnouf C., Thollet G., Fantozzi G., J. Phys. Colloq. Fance 47 (1986) C1-187.

[17] HeidT G., HeImKe G., J. Mater. Sci. 10 (1975) 887. 
[18] Burgess J. F, Neugebaeur C.A., Flanagan G., J. Electrochem. Soc. 122 (1975) 688.

[19] MELlul S., Thèse de l'Université Paris VI (1989).

[20] Tressuer R. E., MoOre T. L., Crane R. L., J. Mater. Sci. 8 (1973) 151.

[21] Mulder C.A.M., Klomp J. T., J. Phys. Colloq. France 46 (1985) C4-111.

[22] TimsitT R. S., WADDINGTON W. G., HuMPhREYS C. J., HuTCHINSON J. L., Ultramicroscopy 18 (1985) 387.

[23] Suganuma K., Okamoto T., Fondamentals of diffusion bonding, Ed. Y. Ishida, Studies Phys. Theor. Chem. (Elsevier) 48 (1987) 71.

[24] Suganuma K., OKamoto T., Shimada M., High Temperatures-High Pressures 16 (1985) 627.

[25] COURBIERE M., KinOSHITA K., KonDOH I., Joining Ceramics, Glass and Metal, Ed. W. Kraft (DGM Informations Gesellshaft, Verlag) 1989.

[26] Shimada M., Suganuma K., OKamoto T., Koizumi M., Reactivity of Solids, Eds. P. Baret et L. C. Dufour (Elsevier) (1985) p. 803.

[27] Yamada T., Kohno A., YOKOI K. and OKADA S., Fondamentals of Diffusion Bonding, Ed. Y. Ishida Studies Phys. Theor. Chem. (Elsevier) 48 (1987) 489.

[28] Iseki T., Kameda T., Maruyama T., J. Mater. Sci. 19 (1984) 1692.

[29] Tremouilles G. et Portier R., J. Phys. Colloq. France 49 (1988) C5-299.

[30] RheE S. K., J. Am. Ceram. Soc. 55 (1972) 300.

[31] NiKOLOPOULOS P., J. Mater. Sci. 20 (1985) 3993.

[32] Livey D. T., Murray P., J. Am. Cer. Soc. 39 (1956) 363.

[33] Chatain D., Rivollet I., Eustathopoulos N., J. Chim. Phys. 83 (1986) 562.

[34] Dufour L. C., PERdREREAU M., Surfaces and Interfaces of Ceramics Metals (Kluwer Academic Publishers, Serie E) 173 (1989) 419.

[35] Chaklader A. C. D., Armstrong A. M., Misra S. K., J. Am. Ceram. Soc. 51 (1968) 630.

[36] O'Brien T. E., Chaklader A. C. D., J. Am. Ceram. Soc. 57 (1974) 329.

[37] Chaklader A. C. D., Gill W. W., Mehrotta S. P., Surfaces and Interfaces in Ceramic and ceramicmetal systems, Eds. J. Pask et A. Evans (Plenum Press N.Y.) 1981, p. 421.

[38] Rivollet I., Chatain D., Eustathopoulos N., Acta Met. 35 (1987) 835.

[39] Jianguo L. I., Coudurier L., ANSARa T., Eustathopoulos N., Ann. Chim. Fr. 13 (1988) 145.

[40] Gillet E., Legressus C., Gillet M., J. Chim. Phys. 84 (1987) 167.

[41] COURBIERE M., Thèse de l'Université Lyon (1987).

[42] HANSEN M., Constitution of binary alloys (McGraw Hill Book Co) (1958) p. 604.

[43] Beraud C., Esnouf C., Courbiere M., Juve M., TreheuX D., J. Mater. Sci. 24 (1989) 4545.

[44] Derby B., WallCa E. R., Met. Sc. 16 (1982) 49.

[45] Goulden D. A., Philos. Mag. 33, (1976) 393.

[46] Ho J. H., VOOK R. W., Philos. Mag. 36 (1977) 1051.

[47] Ishiguro T., Kitazawa H., Mizutani N., Kato M., J. Solid State Chem. 40 (1981) 170.

[48] JACOB K. T. and AlCOCK C. B., J. Am. Ceram. Soc. 58 [5,6] (1975) 192.

[49] STADELManN P. A., Ultramicroscopy 21 (1987) 131.

[50] Ishiguro T., KitaZawa H., Mizutani N., Kato M., J. Solid State Chem. 41 (1982) 132.

[51] POIRIER J. P., Dislocations and Deformation Pastique, Ecole d'Yravals (Editions de Physique) 1979, 223.

[52] BENARD J., L'oxydation des métaux, Tome 1 (Gauthier-Villars et $C^{\text {ie }}$, Paris) (1962) 284.

[53] Paladino A. E., Kingery W. D., J. Chem. Phys. 37 (1962) 957.

[54] Lesage B., Huntz A. M., Scr. Met. 14 (1980) 1143.

[55] Lesage B., Huntz A. M. et Petot-ervas G., Rad. Effects 75 (1983) 283.

[56] Badrour L., Moya E. G., Bernardini J., Moya F., Scr. Met. 20 (1986) 1217.

[57] OISHI Y. et KIngery W. D., J. Chem. Phys. 33 (1960) 480.

[58] Peterson N. L., Wiley C. L., J. Phys. Chem. Solids 45 (1984) 281.

[59] Perinet F., Barbezat S. et Monty C., J. Phys. Colloq. France 41 (1980) C6-315.

[60] Cohen G. et KuCZYNSKi G. C., J. Appl. Phys. 21 (1950) 1339 L.

[61] KirChHEIN R., Acta Met. 25 (1979) 869.

[62] EPICIER T., ESNOUF C., Accepté pour publication à Philos. Mag. Lett.

[63] BERAUD C., EPICIER T., ESNOUF C., Communication acceptee au colloque international CNRS : MDSAM, Aussois (1990). 